\title{
Commissioning with Physics Data at ATLAS
}

\author{
Richard Teuscher* \\ Enrico Fermi Institute, University of Chicago, USA. \\ CERN, Geneva, Switzerland. \\ E-mail: Richard.Teuscher@cern.ch
}

\begin{abstract}
Physics commissioning of the ATLAS detector at the LHC at CERN will proceed in four phases: A first phase with cosmic-ray runs will be dedicated to the initial detector alignment and to calibration. The various systems will be debugged, dead channels will be mapped, etc. Once there is a single beam in the accelerator, beam-halo muons and beam-gas events will allow one to improve upon the initial alignment and calibration. With the first proton-proton collisions, the trigger and detector will be developed, tuning the trigger menus and measuring efficiencies, fake rates, energy scales, resolutions, etc. In the last step, physics channels will be used to commission the detector, and one will start to understand backgrounds to discovery channels. Ideas on what we can learn in each phase, and strategies on how to use the data in practice are presented here. A few examples are given as well of some recent work on the subject.
\end{abstract}

European Physical Society

HEP2005 International Europhysics Conference on High Energy Physics

EPS (July 21 $1^{\text {st }}-27^{\text {th }}$ 2005) in Lisboa, Portugal

* As of January 1, 2006: Dept. of Physics, University of Toronto, Toronto, ON, M5S 1A7, Canada, and CERN, Geneva, Switzerland. 


\section{Introduction}

Commissioning the ATLAS experiment at the LHC at CERN has already begun. A first step was made in 2004, when "slices" of the final subdetector components were exposed to a series of testbeams at CERN. The next milestone was reached in the summer of 2005, when the first cosmic ray muons were observed underground with the ATLAS hadronic Tile calorimeter (TileCal). The next step planned is combined cosmic runs underground, with the liquid argon (LAr) barrel electromagnetic calorimeter together with the TileCal system in the spring of 2006. It is expected that the endcap calorimeters will take cosmic-ray data in the summer and fall of 2006. As the remaining detector components are installed, this programme will lead to a global ATLAS cosmic run in April 2007. This will be followed by the first data with single beams in the LHC and then the first proton-proton collisions starting in the summer of 2007.

As of November 2005, the complete ATLAS barrel toroid magnet system has been installed underground, and the barrel electromagnetic and hadronic calorimeters have been installed and moved to their final position centred at the nominal interaction point. Installation of the muon system is also underway. The inner detectors are being tested in a dedicated surface installation. The main emphasis underground now is on installation of cabling and services, as well as installation of infrastructure and electronics in the two adjacent counting rooms.

\section{Combined Testbeam}

From May-November 2004, a full "slice" of ATLAS was exposed to testbeams in the CERN $\mathrm{H} 8$ and $\mathrm{H} 6$ beamlines. This included components of the inner tracking detectors (pixel system, semi-conducting tracker, and transition-radiation tracker), liquid argon electromagnetic calorimeter, hadronic Tile calorimeter, and the muon system (monitored drift tubes, resistive plate chambers, and cathode-strip chambers). For the first time, all the ATLAS subdetectors, instrumented with final electronics, were integrated and run with a common data-acquisition system. Furthermore, the data collected were analyzed with a common ATLAS software system. Highlights of the combined run include the first pions tracks reconstructed in all 3 tracking systems, the correlation of the z-coordinate of muon tracks in the inner-detector with that of the muon system, correlations of reconstructed energy between the level-1 trigger and calorimeter with a structured $25 \mathrm{~ns}$ beam, and correlations of pion energy loss in the electromagnetic and hadronic calorimeters. The data continue to be analyzed in order to develop calibration techniques for ATLAS, and tune Monte-Carlo simulations.

\section{Cosmic Ray Commissioning}

A milestone in ATLAS commissioning was reached on June 21, 2005, at 18:30, when the first cosmic rays were observed in the ATLAS calorimeter underground. A display of the first event is given in Figure 1. In this illustration, the TileCal, with a pointing geometry, is depicted as a large thick disk, and the energy deposits in its 3 layers are represented as light trapezoids. The trigger configuration in this case was on "back-to-back" muons, from the top to the bottom 
of the calorimeter. The trigger was generated by a custom set of 9U VME coincidence boards, connected to the analogue trigger sums from the calorimeter. Data were read out using final electronics both on the detector and in the underground counting room, and the final offline analysis software was used for event reconstruction. In summary, the full ATLAS chain was exercised from the front-end electronics to the offline software. Based on the success and experience gained in this exercise, a series of further cosmic ray "expert weeks" were followed in the summer and fall of 2005. These will continue in 2006, including combined runs in spring of 2006

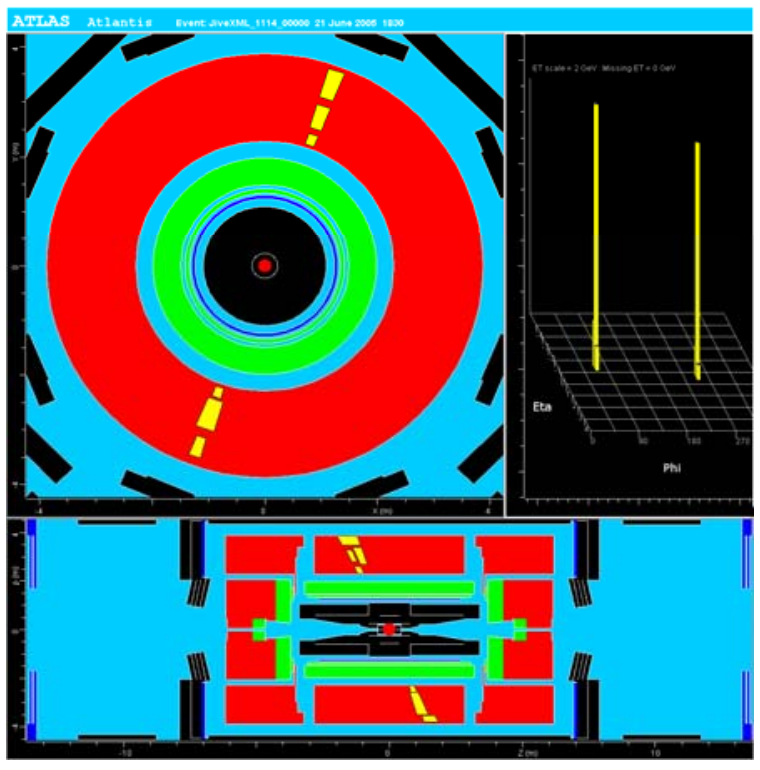

Figure 1: The first cosmic ray in ATLAS with the TileCal and liquid argon barrel calorimeter, and then with the endcap calorimeters in the summer and fall of 2006 .

\section{First LHC beams}

As of November 2005, about 900 of 1232 LHC dipole magnets have been delivered from industry to CERN. The LHC schedule plans for the full 8 accelerator sectors to be cooled down and ready for beam in the summer of 2007. During the first LHC single-beam period, $7 \mathrm{TeV}$ proton beam interactions with rest gas $(\mathrm{H}, \mathrm{C}, \mathrm{O})$ in the imperfect vacuum $\left(3 \times 10^{-8}\right.$ Torr $)$ of the ring near the experiments are expected to produce $\mathrm{O}(100) \mathrm{kHz}$ of beam-gas interactions, with vertices uniform along $\pm 23 \mathrm{~m}$ over $\mathrm{z}$. The products of these interactions will be used to debug the detector components and readout system, check trigger backgrounds, and test offline reconstruction algorithms. Furthermore, beam halo muons, resulting from interactions of the beam with upstream collimators, are predicted to occur at a rate of $\mathrm{O}(100) \mathrm{kHz}$, of which $1 \mathrm{kHz}$ will have an energy above $100 \mathrm{GeV}$. Typically these muons will pass horizontally through the detector, and can be used to check for dead cells, perform energy calibration, and check alignment.

\section{First LHC Collisions}

It is expected that the first pilot run for physics will be in the fall of 2007 , followed by a shutdown and then a long run in 2008. Based on experience with the startup of previous accelerators, such as the SppS and TeVatron Run I, one might expect $\mathrm{O}(1 \%)$ of design luminosity in the initial stages of operation. This would predict an accumulated data set of between 0.1 and $10 \mathrm{fb}^{-1}$ per experiment by the end of 2008 . 
The first data will be used to understand and calibrate the detector, trigger, and software in situ using well-known physics samples. For example, reconstructed decays of $Z \rightarrow$ ee, $\mu \mu$, will be used to commission the tracker, electromagnetic calorimeter, and muon systems. Similarly, due to the high production rate of top pairs (1 per second at 10\% design luminosity) one can study the jet energy scale from the subsequent $\mathrm{W} \rightarrow$ jets decays. The second step will be to understand the basic Standard Model physics at $14 \mathrm{TeV}$ centre-of-mass energy, including the first checks of Monte Carlo simulation, and measuring basic cross-sections, such as minimumbias events, particle multiplicity, QCD jets, $\mathrm{W}, \mathrm{Z}$, and top production. It is expected that the top mass can be determined to $\mathrm{O}(7) \mathrm{GeV}$ with the first data.

The third step will be to measure the backgrounds to new physics, and prepare the road to discovery. Supersymmetry (SUSY) may well be one of the first discoveries beyond the Standard Model at the LHC, due to the large predicted cross-section for squark and gluino production. For example, with $0.1 \mathrm{fb}^{-1}$, squarks and gluinos with masses up to about $1.3 \mathrm{TeV}$ should be detectable, with the sensitivity increasing to over $2 \mathrm{TeV}$ with $10 \mathrm{fb}^{-1}$. The main limitation for a SUSY discovery will most likely not be statistics but rather the understanding of the detectors. Since one of the main signatures of (R-parity conserving) SUSY is missing transverse energy $\left(\mathrm{E}^{\mathrm{T}}{ }_{\text {miss }}\right)$, due to the production of stable neutralinos, backgrounds which fake $\mathrm{E}^{\mathrm{T}}{ }_{\text {miss }}$ will need to be understood. This will include developing cuts against beam-gas, beamhalo, cosmics, events with the $\mathrm{E}_{\text {miss }}^{\mathrm{T}}$ vector along the axis of a jet, and rejecting events with jets in cracks or uninstrumented regions. Further control samples from lower values of $\mathrm{E}^{\mathrm{T}}$ miss, where no SUSY signal is expected, will be used to normalize Monte Carlo distributions to data in order to predict the background in the signal region of higher $\mathrm{E}^{\mathrm{T}}{ }_{\text {miss }}$.

With higher integrated luminosity, of $\mathrm{O}(30) \mathrm{fb}^{-1}$, discovery of the Higgs boson, responsible for generation of particle mass in the Standard Model, will become possible. Since the Higgs mass is at present unknown, beyond the exclusion from LEP of $m_{H}>114 \mathrm{GeV}$, unitarity constraints on WW scattering which require $\mathrm{m}_{\mathrm{H}}<1.2 \mathrm{TeV}$, and fits to precision electroweak data which predict $m_{H}=126+73-48 \mathrm{GeV}$, different decay channels will be studied for a range of possible Higgs masses up to about $1 \mathrm{TeV}$. For example, for light Higgs masses around 130 $\mathrm{GeV}$, the channel $\mathrm{H} \rightarrow \gamma \gamma$ provides the largest expected signal-to-background, which will require excellent calibration of the electromagnetic calorimeters. For Higgs masses above twice the $\mathrm{Z}$ mass, the "golden channel" $\mathrm{H} \rightarrow \mathrm{ZZ} \rightarrow \mu \mu \mu \mu$ will be accessible. By combining these and other channels, a $5 \sigma$ observation of the Higgs boson should be possible in the first years of ATLAS.

\section{Summary}

In conclusion, ATLAS commissioning with physics has already begun, and the detector has given its "first cry" with the observation of cosmic ray muons in the barrel Tile calorimeter during the summer of 2005. Much work lies ahead, in completing the detector, trigger, data acquisition and services installation. The next steps are combined calorimeter cosmic runs in 2006, a global ATLAS cosmic run in spring 2007, and first LHC beam in the summer of 2007. 